\title{
Measurement of the cosmic ray flux near the second knee with the Pierre Auger Observatory
}

\author{
Alan Coleman ${ }^{* a}$ for the Pierre Auger Collaboration ${ }^{b \dagger}$ \\ ${ }^{a}$ Bartol Research Institute, Dept. of Phys. \& Astro., University of Delaware, Newark, DE, USA \\ ${ }^{b}$ Observatorio Pierre Auger, Av. San Martín Norte 304, 5613 Malargüe, Argentina \\ E-mail: auger_spokespersons@fnal.gov \\ Full author list: Pierre Auger Collaboration and additional author(s): \\ http://www.auger.org/archive/authors_icrc_2019_b.html
}

\begin{abstract}
We present the first measurement of the energy spectrum of cosmic rays down to $100 \mathrm{PeV}$ from the Pierre Auger Observatory using the part of the surface-detector array with $750 \mathrm{~m}$ spacing. This analysis is made possible by a set of additional particle triggers, installed in 2013, which lowers the threshold above which the array is fully efficient by half an order of magnitude. The measurement thus covers a critical energy range around $10^{17} \mathrm{eV}$ where previous observations have shown a change in the spectral index, the so-called second or iron knee. The work relies on a geometrical exposure, a nearly $100 \%$ duty cycle, and an almost model-independent analysis, with the energy estimation provided by the fluorescence detector. We discuss the spectrum and the associated uncertainties and compare the results to other studies in the same energy range.
\end{abstract}

36th International Cosmic Ray Conference -ICRC2019-

July 24th - August 1st, 2019

Madison, WI, U.S.A.

\footnotetext{
* Speaker.

${ }^{\dagger}$ for collaboration list see PoS(ICRC2019)1177
} 


\section{Introduction}

The cosmic ray flux spectrum is one of the cornerstone measurements within the field of astroparticle physics. The small irregularities in its shape indicate regions of transition over which the types of particles, classes of sources, propagational effects, etc. are likely changing. Thus, an understanding of the environments in which the highest energy particles in the universe are being accelerated can be directly enhanced by precise measurements of the shape of the energy spectrum.

While the three most notable features of the energy spectrum above $\mathrm{TeV}$ energies are the socalled knee (at $\sim 10^{15.5} \mathrm{eV}$ ), ankle (at $\sim 10^{18.7} \mathrm{eV}$ ), and suppression (at $>10^{20} \mathrm{eV}$ ), other inflection points have been identified through recent high-statistics measurements. Of most interest to this work is the second-knee at roughly $10^{17} \mathrm{eV}$. The second knee corresponds to an observed hardening/softening for light/heavy elements [1-3] and an overall steepening of the all-particle flux [4-9]. This work focuses on the first measurement of the softening of the all-particle spectrum by the Pierre Auger Observatory [10] via an extension to lower energies than previously possible.

\section{Improvements to Cosmic Ray Detection at Auger}

Covering over $3000 \mathrm{~km}^{2}$, Auger is the world's largest cosmic ray detector located in the Mendoza province of Argentina. The observatory's design consists of a hybrid detection scheme which includes both a fluorescence detector (FD) and a surface detector (SD). The FD telescopes are situated on hills at the edge of the observatory and directly image the nitrogen fluorescence light emitted by air molecules which become excited by the developing shower front. In this way, the FD can make nearly calorimetric measurements of the electromagnetic component of air showers and thus set the energy scale for the observatory [11]. On the ground, the secondary particles are observed by the SD which is comprised of around 1600 water-Cherenkov detectors (WCDs), arranged in a hexagonal grid with $1500 \mathrm{~m}$ spacing. Nested within this array is a low-energy extension to the SD which is comprised of identical detectors but with half the grid-spacing, $750 \mathrm{~m}$. The measurements of air showers by the the $750 \mathrm{~m}$ array from January 2014 to August 2018 are the focus of this work.

The acquisition system for the SD [10] relies on a 5-level trigger hierarchy. At the station-level (i.e. for individual WCDs), the T1 and T2 triggers are used to determine when the measured signal in a station is likely the result of air shower particles. Similarly, at the array-level, the triggers (T3, T4, and T5) are used to classify when configurations of T1/T2-triggered stations are consistent with an air shower footprint. A complete description of the triggers can be found in [10].

In 2014, after a decade of SD observations, two new station-level triggers, called Time Over Threshold Deconvolved (TOTd) and Multiplicity of Positive Steps (MoPS), were added to the existing T2 triggers. TOTd and MoPS were designed to be mostly sensitive to the low-energy electromagnetic component of air showers and insensitive to the muons which make up the dominant background for individual WCDs. In practice this is done by identifying the low-amplitude, longduration waveforms which result from electromagnetic particles entering the water and rejecting the short spikes caused by single muons. The inclusion of these triggers into the hierarchy directly increases the SD's sensitivity to the low-energy component of air showers, far from the central axis. The distribution of deposited charge in individual WCDs is shown in the left plot of figure 

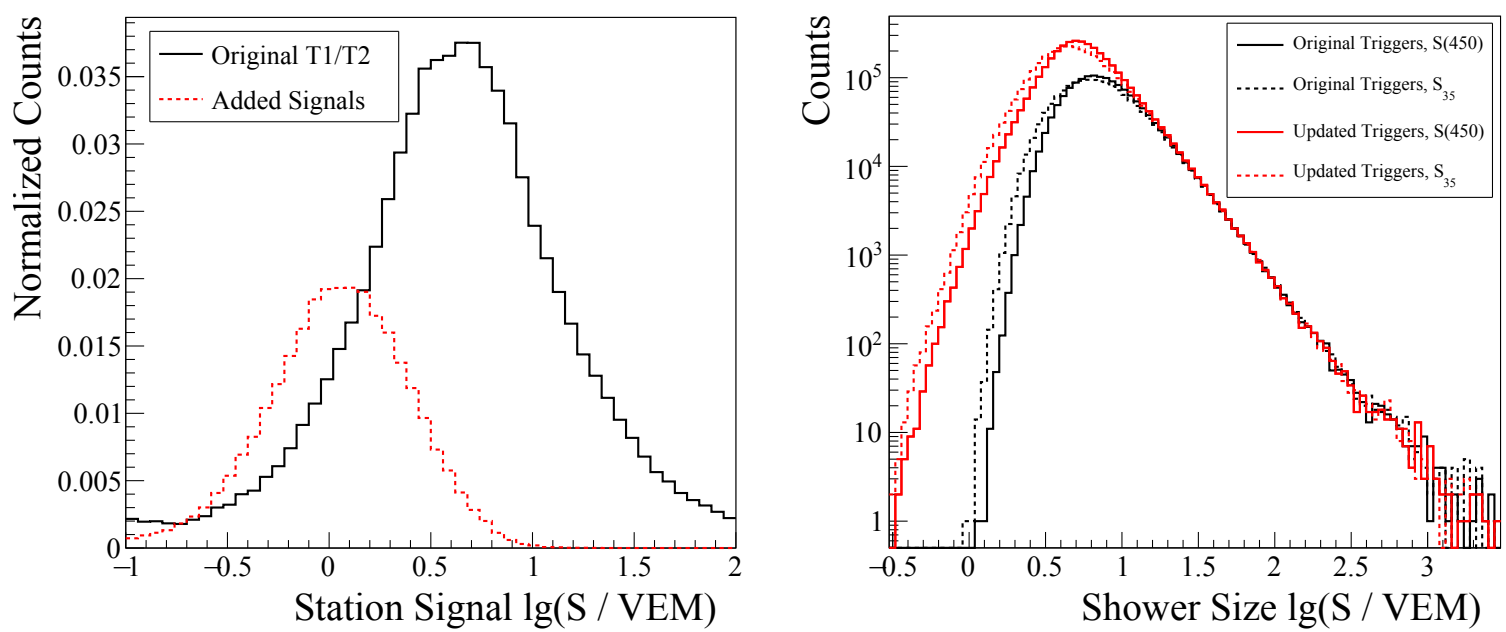

Figure 1: Left: Measured distribution of deposited charge in stations which pass the original T1/T2 algorithms (solid black) and those that pass the new algorithms (red dashed). Right: The observed shower sizes using the full T1/T2 trigger set (red) versus using only the original (black) is shown. Both the raw shower sizes (solid lines) and biascorrected (dashed lines) are included, see text.

1 in units of Vertical Equivalent Muons ${ }^{1}$ (VEM). The signal sizes that can be measured with only the original T1/T2 trigger set is shown in solid black. The dashed red line shows the added signal sizes that are now accessible via the TOTd and MoPS triggers.

The philosophy of the SD reconstruction using the updated trigger set is unchanged with respect to previous SD analyses. First an event cleaning is performed to remove stations which are likely to have been triggered by coincident, background particles. Next, the shower direction is determined using the start times of the signals in the stations. The final step is a fit of the lateral distribution of signals using an empirically chosen lateral distribution function, in this case, a loglog parabola. From this fit, a first-order energy estimator, sometimes referred to as a shower size, is chosen to be the signal that would be expected to be measured at a reference distance ${ }^{2}$ of $450 \mathrm{~m}$ from the shower axis, $S(450)$. For a more detailed description of the reconstruction process, see [13].

Following the reconstruction, two corrections are applied to the $S(450)$ values. The first is an atmospheric correction which accounts for the changing weather conditions at the Observatory. The changes in local air pressure and density affect the measurements of the shower sizes on the ground. Thus, the $S(450)$ values are scaled to what one would expect to observe had the showers developed in an atmosphere with the average pressure and density measured at Auger, see [14].

A second correction is applied to the $S(450)$ values using a constant intensity cut (CIC) [15]. This corrects for the increased attenuation, and thus smaller observed $S(450)$ values, of showers that arrive at higher inclinations. The CIC rescales the energy estimator to that which would have been measured had the shower arrived at a zenith angle of $35^{\circ}, S_{35}$. See [17] for more information.

The increase in sensitivity of the array to lower energy showers can be seen in the right plot of

\footnotetext{
${ }^{1}$ One VEM is defined to be the average amount of signal that would be measured in a station by a muon traversing vertically through the water volume, see [12].

${ }^{2}$ The reference distance was chosen to minimize the impact of the unknown lateral distribution of particles on the energy estimator.
} 

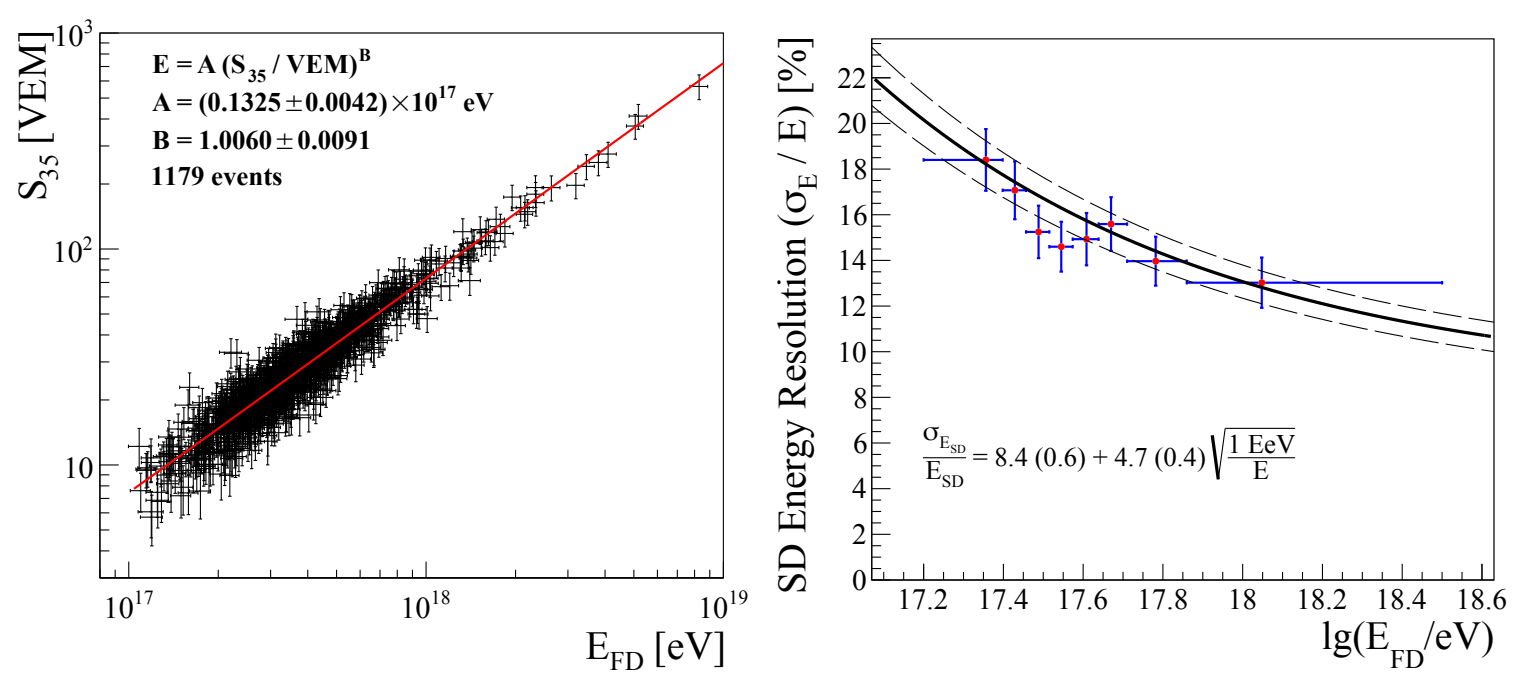

Figure 2: Left: Events used in the energy calibration (black points) are shown along with a power law fit (red line). Right: The SD energy resolution as a function of energy (black line) is plotted with the $1 \sigma$ errors (dashed lines). The resolution in energy bins (red points) are also shown.

figure 1 which shows the distribution of observed shower sizes, $S(450)$ (solid lines) and $S_{35}$ (dashed lines). The shower sizes observed by the $750 \mathrm{~m}$ array when only using the original trigger set is shown in black whereas the sizes now observable after including the TOTd and MoPS algorithms are shown in red. The two distributions agree well for higher energy showers and a clear increase is seen in the number of observed low energy showers now detectable via the new trigger algorithms.

\section{Energy Calibration and Resolution}

To estimate the energy of showers observed by the SD, the $S_{35}$ values are calibrated using the energy measurements from the FD. This is performed using a set of high-quality, golden hybrid, events which were independently reconstructed by both the SD and FD. The relationship between FD energies, $E_{\mathrm{FD}}$, and $S_{35}$ is well described by a power law, $E_{\mathrm{FD}}=A\left(S_{35} / \mathrm{VEM}\right)^{B}$, with fit parameters $A$ and $B$, see [17] for more. The calibration was performed using 1179 golden hybrid events from January 2014 to August 2018. The left plot of figure 2 shows the fitted curve (red line) and the observed shower energies/estimators (black points). The best fit values for the calibration are $A=0.1325 \pm 0.0042 \times 10^{17} \mathrm{eV}$ and $B=1.0060 \pm 0.0091$, a nearly linear relationship.

The same set of golden hybrid events was also used to characterize the energy resolution of the SD. The resolution of the surface array is comprised of two components. The first is a result of the statistical uncertainties in the reconstruction, including contributions from the atmospheric/CIC corrections, which can be estimated on an event-by-event basis. The second component of the SD resolution is the shower-to-shower fluctuations, a result of the stochastic processes that govern the development of particle cascades. Since any two cosmic rays, with identical initial conditions, do not necessarily have the same first interaction depth, the two resultant air showers will arrive at the SD after a differing amount of attenuation, and will not be assigned the same value of $S(450)$. This uncertainty in the shower size cannot be estimated on an event-by-event basis by the SD.

However, using the events observed by both the SD and FD, the combination of these two effects can be estimated. Using the independent energy assignments by the two detectors, the 
distribution of the quantity $z=E_{\mathrm{SD}} / E_{\mathrm{FD}}$ can be calculated for each event, where $\langle z\rangle \equiv 1$, by construction. Thus, $z$ is the ratio of two random variables with a probability distribution, $\operatorname{PDF}(z)$, which depends on the resolution of both the SD, $\sigma_{E, \mathrm{SD}}$, and $\mathrm{FD}, \sigma_{E, \mathrm{FD}}$, and the energy bias between the two detectors. Note that, $\sigma_{E, \mathrm{FD}}$ can be estimated on an event-by-event basis and is typically $8 \%>\frac{\sigma_{E, \mathrm{FD}}}{E_{\mathrm{FD}}}>6.7 \%$ for energies above $10^{17} \mathrm{eV}$ [11]. The distribution of $z$ measurements was fit using $\operatorname{PDF}(z)$ with an energy dependent parameterization of the SD resolution, $\frac{\sigma_{E, S D}}{E_{\mathrm{SD}}}\left(E_{\mathrm{FD}}\right)=\alpha+\beta \sqrt{1 \mathrm{EeV} / E_{\mathrm{FD}}}$, where $\alpha$ and $\beta$ are free parameters. Figure 2 shows the measured resolution for various energy bins (red circles), and the SD's resolution (solid) with 1 sigma error bars (dashed). Above $10^{17.0} \mathrm{eV}$, the SD resolution is $8-24 \%$.

\section{Measurement of the Spectrum}

The event set used to measure the energy spectrum was restricted to the phase space where the array has a $>98 \%$ efficiency to detect an air shower that lands inside the array. This phase space was determined using CORSIKA simulations of air showers for proton and iron primaries using both QGSJetII-04 and EPOS-LHC as hadronic models. For each model-primary combination, $150 \mathrm{k}$ events were simulated, $600 \mathrm{k}$ events in total, with energies ranging from $10^{16} \mathrm{eV}<E_{\mathrm{MC}}<10^{18} \mathrm{eV}$ and zenith angles $0^{\circ}<\theta<55^{\circ}$.

A full detector simulation of the $750 \mathrm{~m}$ array was performed on each event which was then tested against the triggering algorithms. As a function of zenith angle, the triggering efficiency improved slightly at $55^{\circ}$ (the zenith angle cut used in previous $750 \mathrm{~m}$ array analyses). However, a much larger improvement was found if restricting the angular acceptance to $40^{\circ}$. Figure 3 shows the triggering

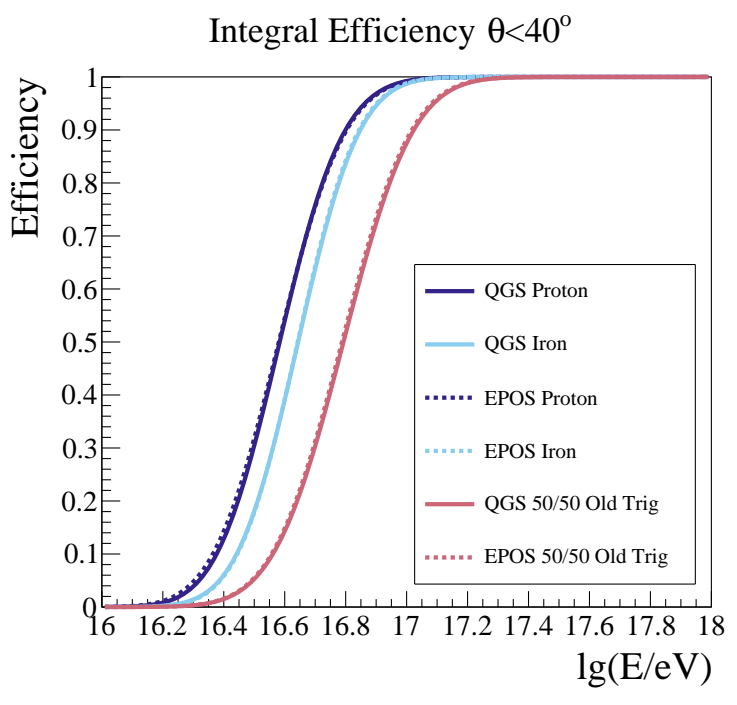

Figure 3: The integral trigger efficiency is shown for showers with zenith angles less than $40^{\circ}$. This quantity is shown for proton (solid lines) and iron (dashed lines) when including the new T1/T2 triggers as well as for a 50$50 \mathrm{mix}$ of proton and iron for only the original trigger set, as a reference.

efficiency at $40^{\circ}$ for all four model-primary combinations as well as for only using the original station triggers, for reference. Note that in this plot, the energy scale is the same as that of the FD, calculated via a cross-calibration to the Monte Carlo energies. For such a zenith angle cut, the array is $>98 \%$ efficient for showers with energies greater than $10^{17} \mathrm{eV}$, regardless of hadronic model or mass.

Two energy spectra were created using data collected from January 2014 to August 2018 by the $750 \mathrm{~m}$ array. The first data set uses a zenith angle and energy cut of $\theta<40^{\circ}$ and $E>10^{17} \mathrm{eV}$, while the second uses the same zenith angle cut as previous $750 \mathrm{~m}$ analyses [16] but a slightly improved energy threshold, $\theta<55^{\circ}$ and $E>10^{17.3} \mathrm{eV}$, as a cross-check. Over this period the 

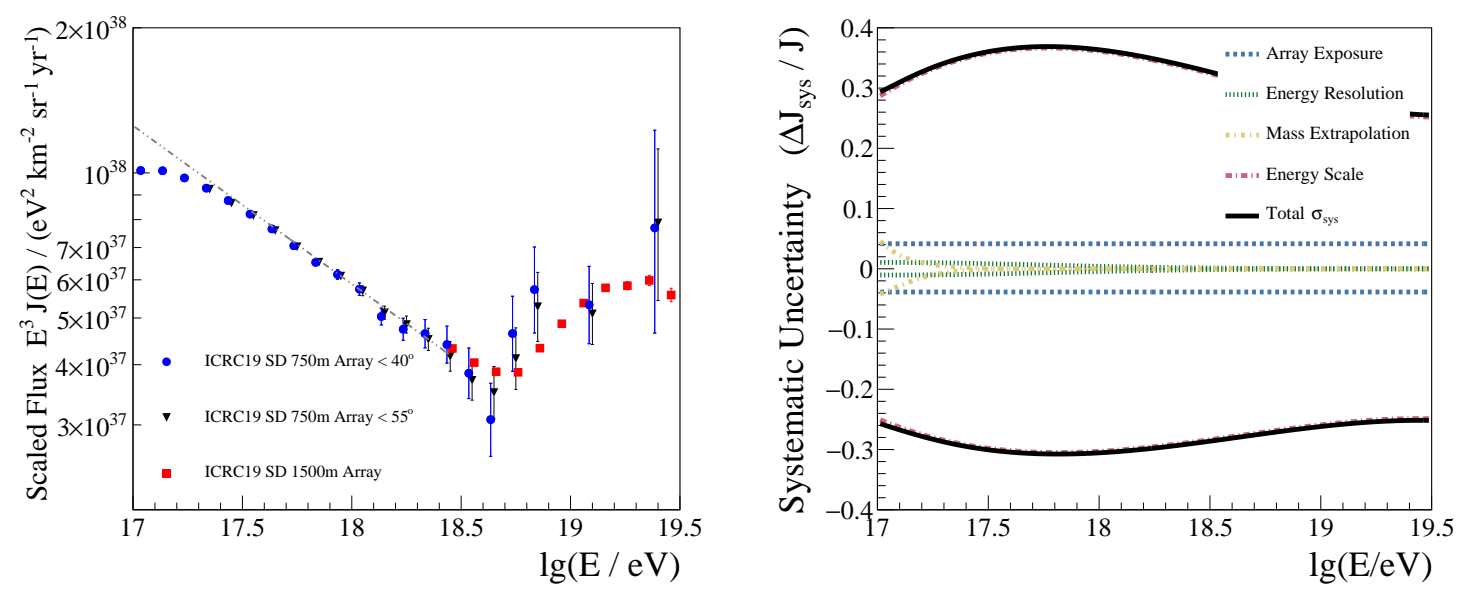

Figure 4: Left: The spectral measurements are shown for the $750 \mathrm{~m}$ array for zenith angle cuts of $\theta<40^{\circ}$ (blue circles) and $\theta<55^{\circ}$ (black triangles) as well as the $1500 \mathrm{~m}$ array (red squares). Right: The systematic uncertainty on the flux is shown as a function of energy.

accumulated exposure was $105.4 \mathrm{~km}^{2} \mathrm{sr} \mathrm{yr}, \sim 570 \mathrm{k}$ events, in the first data set and $171.1 \mathrm{~km}^{2} \mathrm{sr} \mathrm{yr}$, $\sim 190 \mathrm{k}$ events, in the second data set.

A correction was applied to the flux measurements to account for various detector effects that cause events to migrate, asymmetrically, between energy bins. The scaling was calculated using a forward folding method $[16,17]$ which included the previously described measurement of $\sigma_{E, S D}$ and triggering efficiency as well as the energy bias below $10^{17} \mathrm{eV}$ which was determined via Monte Carlo. The resultant spectra for the $40^{\circ}$ (blue circles) and $55^{\circ}$ (black triangles) data sets are shown in the left plot of figure 4 along with the one observed by the $1500 \mathrm{~m}$ array (red squares). All three spectra agree within statistical uncertainties. A more detailed comparison between the $750 \mathrm{~m}$ spectra and those measured by the $1500 \mathrm{~m}$ array and the FD can be found in [17]

This measurement includes a systematic uncertainty that is dominated by the energy scale, $\sigma_{E_{\mathrm{FD}}} / E_{\mathrm{FD}}=14 \%$ which translates to a $\simeq 35 \%$ uncertainty in the flux. The subdominant uncertainties include the array exposure (4\%) and the energy resolution $(<1.5 \%)$. This measurement includes an uncertainty from having to extrapolate the primary-mass composition down to energies below where they have been measured by the FD $\left(E<10^{17.2} \mathrm{eV}\right)$ [18]. However, due to the zenith angle and energy restrictions, the impact on the flux is small $(<5 \%)$. These contributions and the total systematic uncertainty on the flux is shown in the right plot of figure 4.

The flux of cosmic rays in the energy region around $10^{17.0} \mathrm{eV}$ has been probed by many experiments as shown in figure 5. Many of them have observed a softening near this energy which has been called the second knee, including a new Cherenkov measurement using the FD. For Auger, previous measurements by the $\mathrm{SD}$ of the all particle spectrum, restricted to $E>10^{17.5} \mathrm{eV}$, have been consistent with a simple power law below the ankle. However, with the extension of the flux measurement down to $E>10^{17} \mathrm{eV}$, a single power law no longer describes the data well.

To understand the significance of the change in spectral index, $\gamma$, a power law fit, $J(E) \propto E^{-\gamma}$, with $\gamma=3.33$, was extended down to $10^{17} \mathrm{eV}$ (see the dash-dotted gray line in the left plot of figure 4). The residuals with respect to this power law are shown in the left plot of figure 6 (blue circles). The deviation from the power law fit, is as large as $15 \%$ which is well excluded by the statistical 


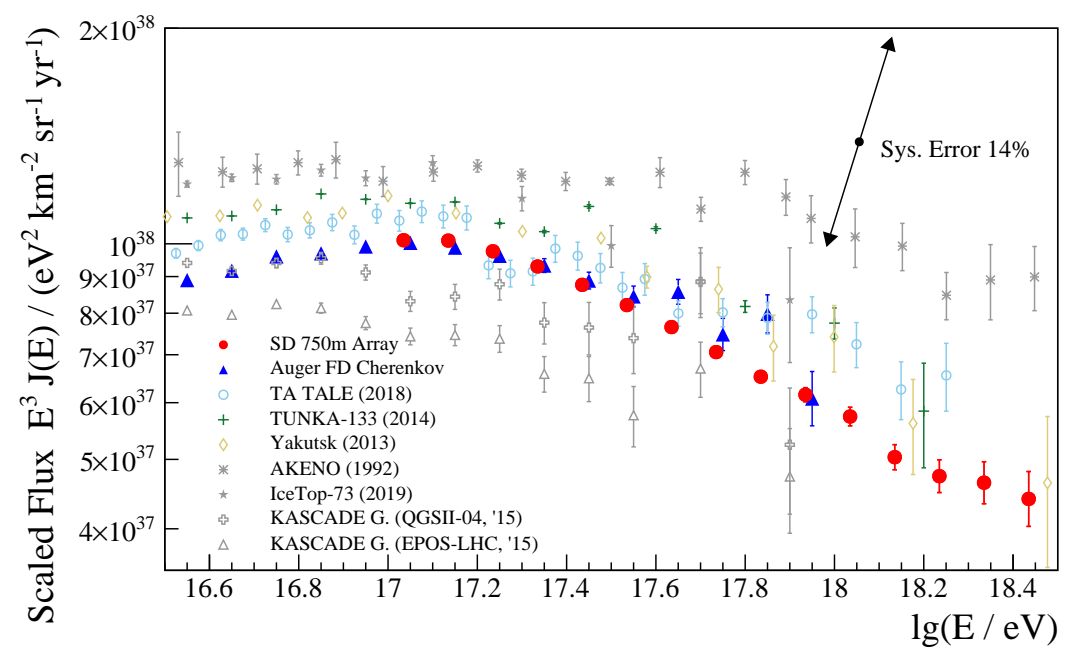

Figure 5: The cosmic ray spectra which observe a softening in the $\sim 10^{17} \mathrm{eV}$ energy region are shown including Auger (both this work and via Cherenkov measurements by the FD [19]), AKENO [4], IceTop [6], KASCADE-Grande [2, 3], TALE [9], TUNKA [8], and Yakutsk [5]. Spectra with energy scales set via calorimetric measurements are shown in color, otherwise, shown in gray.
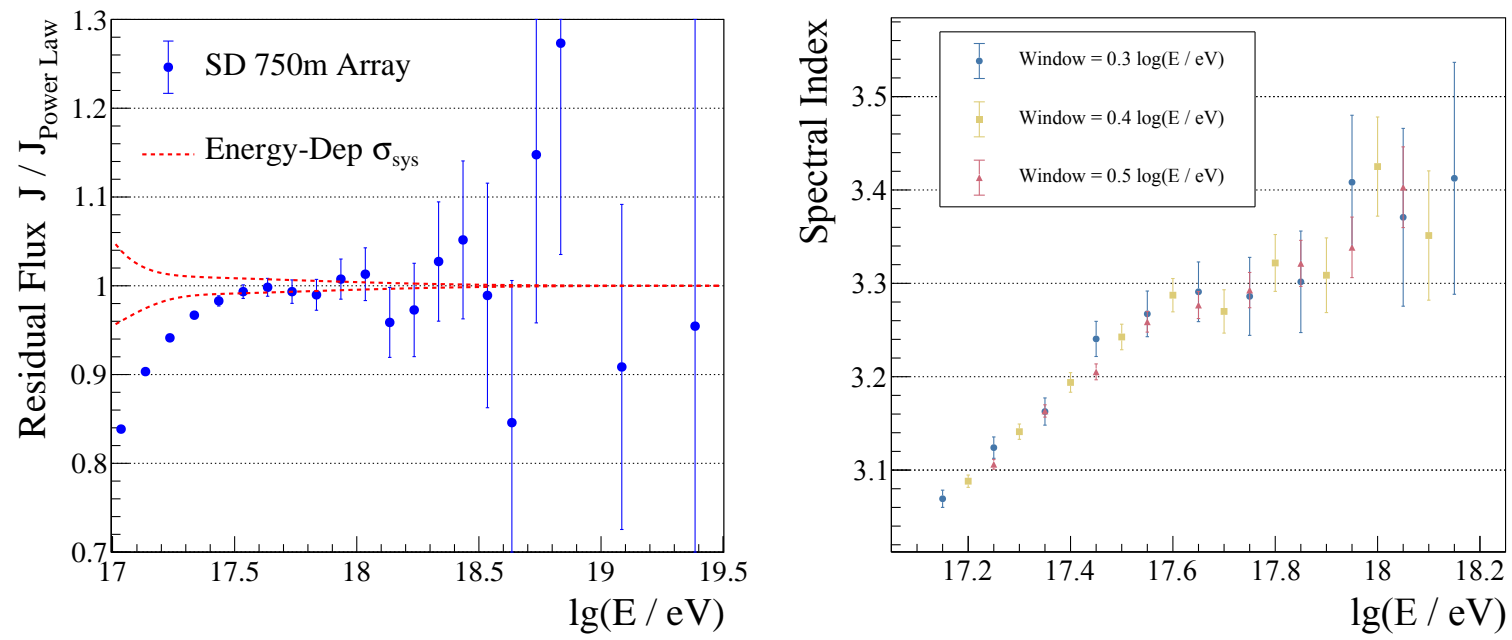

Figure 6: Left: The ratio of the $750 \mathrm{~m}$ array spectrum and a power law (blue points) is shown along with the energydependent systematic uncertainties of the flux. Right: Power law fits to the $750 \mathrm{~m}$ spectrum were performed over limited windows in energy. The spectral indexes are shown for windows of 0.3 (blue circles), 0.4 (gold squares), and 0.5 (red triangles $) \log (E / \mathrm{eV})$.

uncertainties of the measurement. Further, we can rule out such a deviation being a result of a systematic shift by comparing the residuals to the energy-dependent systematic flux uncertainties (red line). The disagreement with respect to a single power law fit below the ankle is at the level of $4.1 \sigma_{\text {sys. }}$.

As a method of estimating the change in spectral index as a function of energy, the spectrum was fit using a series of power laws over limited windows in energy $(0.3,0.4$, and $0.5 \log (E / \mathrm{eV}))$. The spectral indices corresponding to each fit are shown in the right plot of figure 6. A fairly constant increase in $\gamma$ up to about $10^{17.6} \mathrm{eV}$ is observed with a rate of change of about $\frac{d \gamma}{d \log E} \simeq 0.5$. 
Such a change in spectral index is incompatible with a single power law.

\section{Conclusion}

In this work we presented a new measurement of the all-particle cosmic ray spectrum using the $750 \mathrm{~m}$ array at Auger. This work is a significant improvement on previously possible measurements of the flux by the SD in its extension to lower energies, $E>10^{17} \mathrm{eV}$. Such an extension was made possible by an additional set of particle triggers for the WCDs.

Because of the $750 \mathrm{~m}$ array's size, this is the highest precision measurement in an energy region where most other measurements begin to be dominated by statistical uncertainties, including $\sim 570 \mathrm{k}$ events above $10^{17} \mathrm{eV}$. Further, this work is unique in this energy region in that it includes an energy scale set by the FD rather than Monte Carlo simulations and a data-driven approach to estimate the energy resolution. Thus, the result is nearly-model independent. We have shown that there is a significant softening between $10^{17} \mathrm{eV}$ and the ankle where the spectral index increases from $\leq 3.1$ to 3.3. This softening is larger than can be explained by either the statistical or systematic uncertainties of the measurement.

\section{References}

[1] [KASCADE Collaboration], Astropart. Phys. 24.1-2 (2005) 1 [astro-ph / 0505413 ].

[2] [KASCADE-Grande Collaboration], Phys. Rev. Lett. 107.17 (2011) 171104 [astro-ph/1107. 5885].

[3] [KASCADE-Grande Collaboration], Phys. Rev. D 87.8 (2013) 081101 [astro-ph/1304 . 7114 ].

[4] M.Nagano, et al., J. Phys. G Nucl. Partic. 18.2 (1992) 423.

[5] S.P.Knurenko, et al., EPJ Web Conf. 53 (2013) 04004 [ast ro-ph/1112. 2430].

[6] [IceCube Collaboration] Cosmic Ray Spectrum and Composition from PeV to EeV Using 3 Years of Data From IceTop and IceCube, astro-ph/1906.04317, PoS (ICRC2019) 172 (2019).

[7] M.E.Bertaina [KASCADE-Grande Collaboration], in proceedings of The 34th Int. Cosmic Ray Conf., POS (ICRC2015) 359 (2015).

[8] V.V.Prosin, et al., EPJ Web Conf. 121 (2016) 03004.

[9] R.U.Abbasi, et al., Astrophys. J. 865.1 (2018) 74 [astro-ph/1803.01288].

[10] A.Aab [Pierre Auger Collaboration], Nucl. Instrum. Meth. A 798 (2015) 172 [astro-ph/1502.01323].

[11] B.Dawson [Pierre Auger Collaboration], in proceedings of The 36th Int. Cosmic Ray Conf., POS ( ICRC2019) 231 (2019).

[12] X.Bertou, et al., Nucl. Instrum. Meth. A 568.2 (2006) 839.

[13] D.Mockler [Pierre Auger Collaboration], in proceedings of The 36th Int. Cosmic Ray Conf., POS ( ICRC2019) 353 (2019).

[14] A.Aab [Pierre Auger Collaboration], JINST 12 (2017) P02006

[15] J.Hersil, et al., Phys. Rev. Lett. 6 (1961) 22.

[16] F. Fenu [Pierre Auger Collaboration], in proceedings of The 35th Int. Cosmic Ray Conf., Po.S ( ICRC2017) 486 (2017).

[17] V.Verzi [Pierre Auger Collaboration], in proceedings of The 36th Int. Cosmic Ray Conf. PoS ( ICRC2 019$) 450$ (2019).

[18] J.Bellido [Pierre Auger Collaboration], in proceedings of The 35th Int. Cosmic Ray Conf., POS (ICRC2017) 506 (2017)

[19] V.Novotny [Pierre Auger Collaboration], in proceedings of The 36th Int. Cosmic Ray Conf., POS ( ICRC2019) 374 (2019). 